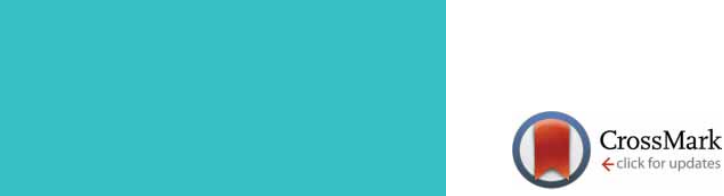

\title{
Anxiety disorders and asthma among adolescents in Uganda: role of early-life
} exposures

\author{
Harriet Mpairwe (i] ${ }^{1,2}$, Richard Stephen Mpango ${ }^{1}$, Wilber Sembajjwe ${ }^{1}$, \\ Emily L. Webb ${ }^{2}$, Alison M. Elliott ${ }^{1,2}$, Neil Pearce ${ }^{2}$ and Eugene Kinyanda ${ }^{1}$
}

Affiliations: ${ }^{1}$ Medical Research Council/Uganda Virus Research Institute and London School of Hygiene and Tropical Medicine Uganda Research Unit, Entebbe, Uganda. ${ }^{2}$ London School of Hygiene and Tropical Medicine, London, UK.

Correspondence: Harriet Mpairwe, MRC/UVRI Uganda Research Unit On AIDS - Immunomodulation and Vaccines Programme, Plot 51-59, Nakiwogo Road, Box 49, Entebbe, Uganda.

E-mail: Harriet.Mpaiwedmrcuganda.org

ABSTRACT The reasons for the positive association between anxiety disorders and asthma are unknown. We investigated the possible role of shared exposures in early life.

We conducted a case-control study among adolescents (age 12-17 years) with and without asthma in urban Uganda, as part of a larger asthma case-control study. Anxiety disorders were diagnosed by psychiatric clinical officers. We focused on generalised anxiety disorder (GAD), panic disorder and social anxiety disorder. Asthma was doctor-diagnosed by study clinicians. We used questionnaires to collect data on early-life exposures. The data were analysed using multiple logistic regression.

We enrolled 162 adolescents; 73 of them had asthma. Adolescents with asthma were more likely to have any of the three anxiety disorders studied (46.6\%) than adolescents without asthma (21.4\%) (adjusted OR (aOR) 2.68, 95\% CI 1.30-5.53). The association was strong for GAD (aOR 4.49, 95\% CI 1.48-13.56) and panic disorder (aOR 5.43, 95\% CI 2.11-14.02), but not for social anxiety disorder. The early-life risk factors associated with anxiety disorders among adolescents were similar to asthma risk factors previously published, including urban residence at birth (aOR 3.42, 95\% CI 1.29-9.09) and during most of the first 5 years of life (aOR 2.87, 95\% CI 1.07-7.66), father's tertiary education (aOR 2.09, 95\% CI 1.00-4.37), and adolescent's history of other allergy-related diseases (aOR 4.64, 95\% CI 1.66-13.00).

We confirm a positive association between anxiety disorders and asthma among adolescents in urban Uganda. The early-life risk factors associated with anxiety disorders among adolescents were similar to those for asthma in the same age group, suggesting shared underlying environmental exposures.

@ERSpublications

In this study in Uganda, adolescents with anxiety disorders were more likely to have asthma. Both conditions had similar risk factors in early life, including urban residence and higher parental education, suggesting shared underlying exposures. https://bit.ly/3rCtcPa

Cite this article as: Mpairwe H, Mpango RS, Sembajjwe W, et al. Anxiety disorders and asthma among adolescents in Uganda: role of early-life exposures. ERJ Open Res 2021; 7: 00749-2020 [https://doi.org/10.1183/23120541.00749-2020].

This article has supplementary material available from openres.ersjournals.com

Received: 13 Oct 2020 | Accepted after revision: 5 March 2021

Copyright $\odot$ The authors 2021. This version is distributed under the terms of the Creative Commons Attribution Licence 4.0. 


\section{Introduction}

Anxiety disorders are among the first psychiatric disorders to emerge in childhood and adolescence $[1,2]$, and are the sixth leading cause of illness and disability among adolescents aged 10-14 years and the ninth for adolescents aged 15-19 years globally [1]. Anxiety disorders are common, with global estimates for lifetime prevalence of $9.9-16.7 \%$ (interquartile range) [3]. Anxiety disorders, as classified by the Diagnostic and Statistical Manual of Mental Disorders, Fifth Edition (DSM-5), include generalised anxiety disorder (GAD), social anxiety disorder, separation anxiety disorder, specific phobia and panic disorder [2]. The causes of anxiety disorders are not fully known, but environmental, developmental and biological risk factors in early life have been identified [2]. Adolescents are at greater risk of anxiety disorders if they have chronic illnesses, such as asthma [1].

Asthma is the most common non-communicable disease among children and is estimated to affect 235 million people globally, yet we still do not fully understand the causes [4]. Several early-life risk factors for asthma have been suggested, including parental asthma, prenatal environmental tobacco smoke exposure and prematurity [5]. It has been observed that in African and other low- and middle-income countries (LMICs), the prevalence of asthma is higher in urban than rural areas [6-9]. Our study in Uganda found that the risk of asthma depended on area of residence at birth and in the first 5 years of life: lowest risk for rural-born children, with risk doubling among town-born children and trebling among city-born children [10]. This and other studies highlight the importance of environmental exposures in early life for the development of asthma in later childhood [5, 10, 11].

Asthma and anxiety disorders are positively associated among children, adolescents and adults [12, 13]. The reasons for this have not been fully resolved, resulting in an ongoing "chicken and egg" debate about which comes first, with some authors suggesting a bidirectional relationship $[14,15]$. In addition, a third alternative to possibly explain the positive association between anxiety disorders and asthma has been suggested as shared liability, through shared environmental exposures [16]. There is a scarcity of data on anxiety disorders and asthma among adolescents in sub-Saharan Africa. We investigated the associations between anxiety disorders and asthma among adolescents in urban Uganda, and the associations between reported exposures in early life and anxiety disorders in adolescents.

\section{Methods}

The data that support the findings of this study are available in the London School of Hygiene and Tropical Medicine Data Compass digital repository [17]. We report our findings according to the STROBE (Strengthening the Reporting of Observational Studies in Epidemiology) guidelines [18].

\section{Study design and enrolment procedures}

This study was nested within a larger asthma case-control study that has previously been reported [10]. Briefly, the parent case-control study enrolled schoolchildren (age 5-17 years), with and without asthma, from schools in Wakiso District, an urban area in central Uganda. The larger study was conducted from May 2015 to July 2017, and aimed to recruit all schoolchildren with asthma and a random sample of two non-asthma controls for each case. All participants in the larger study provided voluntary informed consent for additional information regarding their psychological wellbeing. For 6 months only (March to August 2016), a team of two experienced psychiatric clinical officers joined the larger study team to enrol participants in this current study. The psychiatric clinical officers collected data on anxiety disorders from adolescents (age 12-17 years) on the day they enrolled in the larger case-control study. This additional data collection involved answering questions to diagnose anxiety disorders (see next section). The psychiatric clinical officers were supervised by a clinical psychologist and psychiatrist. We defined adolescents as children 12-17 years of age (the World Health Organization definition is 10-19 years [19]); 12 years was the required minimum age for the key diagnostic tool for anxiety disorders (see next section) and 17 years was the upper age limit for the parent case-control study [10].

\section{Definition of anxiety disorders}

We used the DSM-5-referenced instrument, the Youth's Inventory-4R (YI-4R), to diagnose anxiety disorders [20]. The Y1-4R is a youth-reported scale that provides invaluable insight into how youth perceive their problems. The symptom/sign and impairment items, and how these were scored to generate the diagnostic categories, as well as the reliability and validity of this scale among young people in Uganda have been reported previously [21]. Here, we focused on three anxiety disorders whose onset typically occurs during adolescence: GAD, panic disorder and social anxiety disorder [2]. On average, assessment with the YI-4R scale lasted for $\sim 45 \mathrm{~min}$. The participation rate of adolescents in the current study was determined by the rate of data collection of the two psychiatric clinical officers, since enrolment to both the larger and current study took place on the same day. The current study aimed for a case:control ratio of 1:1, while the larger study aimed for a 1:2 ratio. Recruitment rates were similar across all schools. 
Any adolescents who required psychiatric management received initial attention from the psychiatric clinical officers and were referred for further management, appropriately.

\section{Asthma diagnosis and control}

Adolescents were screened for asthma using the International Study of Asthma and Allergies in Childhood questionnaire [22]. Adolescents with a history of wheezing in the last 12 months underwent a clinical assessment by study clinicians which involved a detailed medical and treatment history to diagnose asthma [10]. In addition, we assessed asthma control in the last 4 weeks using the childhood Asthma Control Test (cACT) [23] for 12-year-old adolescents and the Asthma Control Test (ACT) [24] for those aged $\geqslant 13$ years. Adolescents without a history of wheezing or any asthma symptoms were enrolled as non-asthma controls [10].

\section{Additional data collected}

We used questionnaires to collect data from parents or guardians on potential early-life risk factors such as area of residence at the time of birth (proxy for residence in pregnancy), main area of residence at 0-5 years of age for the adolescent, parental education and history of allergy. We used questionnaires to collect data from adolescents on what they thought/had noticed triggered their asthma symptoms. We used the question "What triggers seem to make your asthma symptoms worse?" and provided a list of putative triggers (based on our pilot study), including exercise, chest infection, air pollution/irritants, cold air, dust, psychological distress/laughter, pollens/pets, do not know and other (specify). Adolescents responded with either "Yes", "No" or "Not sure" for each trigger and reported any other triggers not on the list. We performed skin prick tests (SPTs) for allergic sensitisation using seven crude extracts (Dermatophagoides mix of Dermatophagoides farinae and Dermatophagoides pteronyssinus, Blomia tropicalis, Blattella germanica, peanut, cat, pollen mix of weeds, mould mix of Aspergillus species; ALK-Abelló, Hørsholm, Denmark), as well as a saline negative control and histamine positive control [25]. We used the standard procedures for SPTs as previously described [10, 25].

\section{Ethical considerations}

Adolescents provided written informed assent and their parents or guardians provided written informed consent. The consenting process was conducted in either English or Luganda (the main language in the study area). The study was conducted according to Good Clinical Practice guidelines and obtained ethical approval from two independent bodies: Uganda Virus Research Institute Research and Ethics Committee (reference GC/127/14109/481) and Uganda National Council for Science and Technology (reference HS 1707).

\section{Statistical methods}

Data were collected on pre-coded paper questionnaires and double entered in OpenClinica open source software version 3.1.4 (OpenClinica LLC and collaborators, Waltham, MA, USA). Data were analysed in Stata version 15 (StataCorp, College Station, TX, USA).

We used multivariable logistic regression to estimate odds ratios and 95\% confidence intervals. We adjusted for confounders such as age and sex determined a priori, and father's highest education status, as suggested in the literature to be strongly associated with socioeconomic status [26]. We did not additionally adjust for mother's education since this was strongly associated with father's education, to avoid collinearity. We explored risk factors for anxiety disorders based on determinants associated with asthma from the larger study, since we aimed to investigate whether there were shared early-life exposures for the two conditions. Multiple linear regression models were fitted for ACT scores and adjusted for the confounders as described earlier. For the purpose of this analysis, the outcomes in the regression models varied for the different analyses: for the association between anxiety disorders and asthma and with asthma control, asthma and asthma control scores were considered the outcome; for the association between early-life risk factors and anxiety disorders, anxiety disorders were considered the outcome. We did not impute missing data.

\section{Results}

The details of the numbers of participants enrolled in the parent case-control study have been described elsewhere [10]. Briefly, 1702 schoolchildren were enrolled, 562 with and 1140 without asthma, from 55 schools in Wakiso. This current study enrolled 162 adolescents. The mean age was 14.3 years (range 12-17 years) and 111 (68.5\%) of these were girls (girls were $55.9 \%$ in the parent case-control study). Of them, 73 adolescents had asthma. 


\section{Anxiety disorders and asthma}

The proportion of children reporting anxiety disorders was different for adolescents with and without asthma; the overall prevalence of any of the three anxiety disorders studied was higher (46.6\%) among adolescents with asthma than among adolescents without asthma (21.4\%) (adjusted OR (aOR) 3.05, 95\% CI 1.50-6.21) (table 1). This difference was particularly strong for GAD (aOR 5.16, 95\% CI 1.71-15.55) and panic disorder (aOR 5.92, 95\% CI 2.31-15.19), but not for social anxiety disorder (aOR 1.68, 95\% CI 0.74-3.84) (table 1).

In order to investigate further the hypothesis that both anxiety disorders and asthma may share early-life exposures, we additionally adjusted for urban residence at birth in the regression model assessing the association between anxiety disorders and asthma: we saw a reduction in effect size in the newly adjusted odds ratios; compared with values in table 1 (column 5), the newly adjusted odds ratios reduced to aOR 2.68 (95\% CI 1.30-5.53) for any of the three anxiety disorders, aOR 4.49 (95\% CI 1.48-13.56) for GAD, aOR 5.43 (95\% CI 2.11-14.02) for panic disorder and aOR 1.46 (95\% CI 0.63-3.37) for social anxiety disorder (table 1, column 6).

\section{Anxiety disorders and asthma control}

ACT scores were generated from the CACT and ACT [27]. Asthma control was poorer among adolescents with any of the three anxiety disorders, as depicted by the lower ACT scores (adjusted mean difference -2.66 , 95\% CI -5.01--0.30) (table 2). A similar pattern was observed for all the individual anxiety disorders, but the differences were not statistically significant (table 2).

\section{Risk factors associated with anxiety disorders}

We found that adolescents with anxiety disorders, compared with their counterparts without, were more likely to have been born in an urban area (versus rural: aOR 3.42, 95\% CI 1.29-9.09), raised in an urban area for their first 5 years of life (aOR 2.87, 95\% CI 1.07-7.66), have fathers with tertiary education (versus secondary or less: aOR 2.09, 95\% CI 1.00-4.37), have a father with a reported history of asthma (aOR 6.52, 95\% CI 1.59-26.76) and report a history of other allergy-related diseases (rhinitis, allergic conjunctivitis or eczema: aOR 4.64, 95\% CI 1.66-13.00), but there was no difference in allergic sensitisation (SPT to any of seven allergens: aOR 1.31, 95\% CI 0.65-2.67) (table 3). These risk factors were similar to the asthma risk factors we reported from the parent case-control study [10].

Given the observed finding of residence in early life, father's education status and adolescent's asthma as risk factors for anxiety disorders, we explored how the different combinations of risk factors were associated with anxiety disorders. First, compared with adolescents born in rural areas and whose fathers had only primary or secondary education (reference group), adolescents born in urban areas and whose fathers had a tertiary education had the highest risk of anxiety disorders (aOR 8.67, 95\% CI 2.14-35.10) (supplementary table S1). Second, compared with adolescents born in rural areas and without asthma (reference group), adolescents born in urban areas and with asthma had the highest risk for anxiety disorders (aOR 6.03, 95\% CI 1.81-20.02) (supplementary table S2).

\section{TABLE 1 Anxiety disorders among adolescents with and without asthma in Uganda ${ }^{\#}$}

Asthma cases Non-asthma controls Crude OR

$[95 \% \mathrm{Cl}]$

Subjects
Any of three anxiety disorders
No (109)
Yes (53)
Generalised anxiety disorder
No (141)
Yes (21)
Panic disorder
No (130)
Yes (32)
Social anxiety disorder
No (131)
Yes (31)

89

73

$\begin{array}{cc}39(53.4) & 70(78.6) \\ 34(46.6) & 19(21.4) \\ & \\ 57(78.1) & 84(94.4) \\ 16(21.9) & 5(5.6) \\ & \\ 49(67.1) & 81(91.0) \\ 24(32.9) & 8(9.0) \\ 55(75.3) & 76(85.4) \\ 18(24.7) & 13(14.6)\end{array}$

Adjusted OR
(95\% CI)

Adjusted $\mathrm{OR}^{+}$ (95\% CI)

p-value

Data are presented as $n$ or $n(\%)$, unless otherwise stated. ${ }^{\#}: n=162 ;$ ": adjusted for child's age, sex and father's highest education level attained; ${ }^{+}$: adjusted for child's age, sex, father's highest education level attained and child's residence at birth. 
TABLE 2 Anxiety disorders and asthma control among adolescents with asthma in Uganda ${ }^{\ddagger}$

\begin{tabular}{|c|c|c|c|c|}
\hline & \multicolumn{4}{|c|}{ ACT scores" } \\
\hline & \multicolumn{2}{|c|}{ Univariate analysis } & \multicolumn{2}{|l|}{ Multivariate analysis $^{+}$} \\
\hline & Mean士sD & p-value & Adjusted mean difference $(95 \% \mathrm{CI})$ & p-value \\
\hline \multicolumn{5}{|c|}{ Any of three anxiety disorders } \\
\hline No (38) & $21.24 \pm 5.17$ & & Reference & \\
\hline Yes (34) & $18.26 \pm 4.93$ & 0.01 & $-2.66(-5.01--0.30)^{\S}$ & 0.03 \\
\hline \multicolumn{5}{|c|}{ Generalised anxiety disorder } \\
\hline No (56) & $20.48 \pm 5.40$ & & Reference & \\
\hline Yes (16) & $17.56 \pm 3.98$ & 0.05 & $-2.34(-5.20-0.52)$ & 0.11 \\
\hline \multicolumn{5}{|c|}{ Panic disorder } \\
\hline No (48) & $20.77 \pm 5.47$ & & Reference & \\
\hline Yes (24) & $17.96 \pm 4.26$ & 0.03 & $-2.06(-4.70-0.58)$ & 0.12 \\
\hline \multicolumn{5}{|c|}{ Social anxiety disorder } \\
\hline No (54) & $20.24 \pm 5.02$ & & Reference & \\
\hline Yes (18) & $18.61 \pm 5.84$ & 0.26 & $-1.39(-4.21-1.43)$ & 0.33 \\
\hline \multicolumn{5}{|c|}{$\begin{array}{l}\text { Data are presented as } n \text {, unless otherwise stated. ACT: Asthma Control Test. }{ }^{\#}: n=72 ;{ }^{~}: \text { based on the } \\
\text { childhood ACT and ACT (scores ranged from } 8 \text { to } 34 \text {, low scores indicating poor asthma control and high } \\
\text { scores indicating well controlled asthma); }{ }^{+} \text {: final models adjusted for child's age, sex and father's highest } \\
\text { education level attained; } \$ \text { : adjusted linear regression model constant }=36.05, R^{2}=0.21, F(5,66)=3.46 \text {, } \\
p=0.008, n=72 \text {. }\end{array}$} \\
\hline
\end{tabular}

\section{Psychological triggers for asthma and residence in early life}

Given the positive association between anxiety disorders and urban residence in early life, we conducted an exploratory analysis to investigate the association between reporting psychological triggers for asthma and area of residence in early life, among all adolescents with asthma enrolled in the parent case-control study ( $n=275)$ [27], of whom the 162 participants with data on anxiety disorders comprised a subset.

We found a weak positive trend between adolescents with psychological triggers and urban residence at birth. There was a trend towards statistical significance between psychological triggers and urban residence in the first 5 years of life: in a town ( $\mathrm{aOR} 2.28$, 95\% CI $0.88-5.86$ ) or the city (aOR 3.53, 95\% CI $1.12-11.18)\left(\mathrm{p}_{\text {trend }}=0.03\right)$ (table 4$)$.

\section{Discussion}

We observed a positive association between anxiety disorders and asthma among adolescents in urban Uganda. We also found that adolescents with both asthma and anxiety disorders had poorer asthma control than asthmatic adolescents without anxiety disorders. Additionally, early-life risk factors associated with anxiety disorders among adolescents were similar to risk factors for asthma that we described previously, including urban residence in early life (compared with rural), parental tertiary education (compared with secondary or lower education), father's (and mother's) reported history of asthma and adolescents' own history of other allergy-related diseases [10]. The positive associations between anxiety disorders and asthma among adolescents were of similar magnitude to those previously reported from high-income countries $[12,13]$. The strong association between asthma and two anxiety disorders, i.e. GAD and panic disorder, but not with social anxiety disorder, is unlikely due to the study design, but probably due to differences in the characteristics of anxiety spectrum disorders.

Although the temporal relationship between anxiety disorders and asthma is not yet clearly understood, this study has demonstrated that both conditions share risk factors in early life. Of particular interest was the observation that the risk of anxiety disorders among adolescents in urban Uganda was related to their residence in early life, with the highest risk among adolescents born and raised in urban areas. This is similar to what we described for asthma previously: compared with schoolchildren in urban Uganda who were born and raised rural areas, the risk of asthma was double among adolescents born and raised in any town and triple among those born and raised in the city [10]. Indeed, schoolchildren born and raised in urban areas whose parents had a tertiary education seem to be at a higher risk of both conditions. These results suggest that the positive association between anxiety disorders and asthma during adolescence may have its roots in early life, due to shared environmental risk factors in urban areas, different from exposures in rural areas. This hypothesis of shared environmental factors in early life is also supported by twin studies from Europe that have shown familial aggregation of both anxiety disorders and asthma among children, which could not be explained by genetic factors [16]. 


\begin{tabular}{|c|c|c|c|c|c|}
\hline & $\begin{array}{l}\text { With } \\
\text { anxiety }\end{array}$ & $\begin{array}{l}\text { Without } \\
\text { anxiety }\end{array}$ & $\begin{array}{l}\text { Crude OR } \\
(95 \% \mathrm{CI})\end{array}$ & $\begin{array}{l}\text { Adjusted OR } \\
(95 \% \mathrm{Cl})^{\pi}\end{array}$ & p-value \\
\hline Subjects & 53 & 109 & & & \\
\hline \multicolumn{6}{|l|}{$\begin{array}{l}\text { Adolescent's residence at } \\
\text { birth }\end{array}$} \\
\hline Rural (38) & $6(11.3)$ & $32(29.4)$ & 1 & 1 & \\
\hline Urban (124) & 47 (88.7) & $77(70.6)$ & 3.25 (1.27-8.37) & 3.42 (1.29-9.09) & 0.01 \\
\hline \multicolumn{6}{|l|}{$\begin{array}{l}\text { Adolescent's residence } \\
\text { during most of } 0-5 \text { years }\end{array}$} \\
\hline Rural (35) & $6(11.3)$ & $29(26.6)$ & 1 & 1 & \\
\hline Urban (127) & 47 (88.7) & $80(73.4)$ & $2.84(1.10-7.34)$ & $2.87(1.07-7.66)$ & 0.04 \\
\hline \multicolumn{6}{|l|}{ Father's education status } \\
\hline Secondary or less (119) & $34(64.1)$ & $85(78.0)$ & 1 & 1 & \\
\hline Tertiary (43) & 19 (35.9) & $24(22.0)$ & $1.98(0.96-4.07)$ & $2.09(1.00-4.37)$ & 0.05 \\
\hline \multicolumn{6}{|l|}{ Father's history of asthma ${ }^{+}$} \\
\hline None (136) & 40 (83.3) & $96(97.0)$ & 1 & 1 & \\
\hline Yes (11) & $8(16.7)$ & $3(3.0)$ & $6.40(1.61-25.37)$ & $6.52(1.59-26.76)$ & 0.005 \\
\hline \multicolumn{6}{|l|}{$\begin{array}{l}\text { Adolescent's history of } \\
\text { allergy-related diseases } \\
\S\end{array}$} \\
\hline None (39) & $5(9.4)$ & $34(31.2)$ & 1 & 1 & \\
\hline Yes (123) & $48(90.6)$ & 75 (68.8) & 4.35 (1.59-11.90) & $4.64(1.66-13.00)$ & 0.001 \\
\hline \multicolumn{6}{|l|}{$\begin{array}{l}\text { Adolescent's positive SPT to } \\
\text { any of seven allergens }{ }^{f}\end{array}$} \\
\hline None (101) & $32(60.4)$ & $69(63.9)$ & 1 & 1 & \\
\hline Yes (60) & $21(39.6)$ & $39(36.1)$ & $1.16(0.59-2.28)$ & $1.31(0.65-2.67)$ & 0.45 \\
\hline
\end{tabular}

Data are presented as $n$ or $n(\%)$, unless otherwise stated. SPT: skin prick test. \#: $n=162$ (missing data: father's history of asthma $n=15$ and adolescent's positive SPT $n=1$ ); ๆ: adjusted for age, sex and father's education level; ${ }^{+}$: similar trend for mother's history of asthma, albeit small numbers; ${ }^{\S}$ : adolescent's history of any of rhinitis, allergic conjunctivitis and eczema; $f$ : allergens were crude extracts of Dermatophagoides mix, Blomia tropicalis, Blattella germanica, peanut, cat, pollen mix of weeds and mould mix of Aspergillus species.

Several studies in LMICs have consistently found a higher prevalence of asthma among children and adults in urban than rural areas, but the specific causative environmental exposures have not been identified [9]. It is likely that several environmental factors are at play, and initial ecological studies have reported that

TABLE 4 Area of residence in early life and reported psychological triggers for asthma symptoms among adolescents with asthma in urban Uganda ${ }^{\#}$

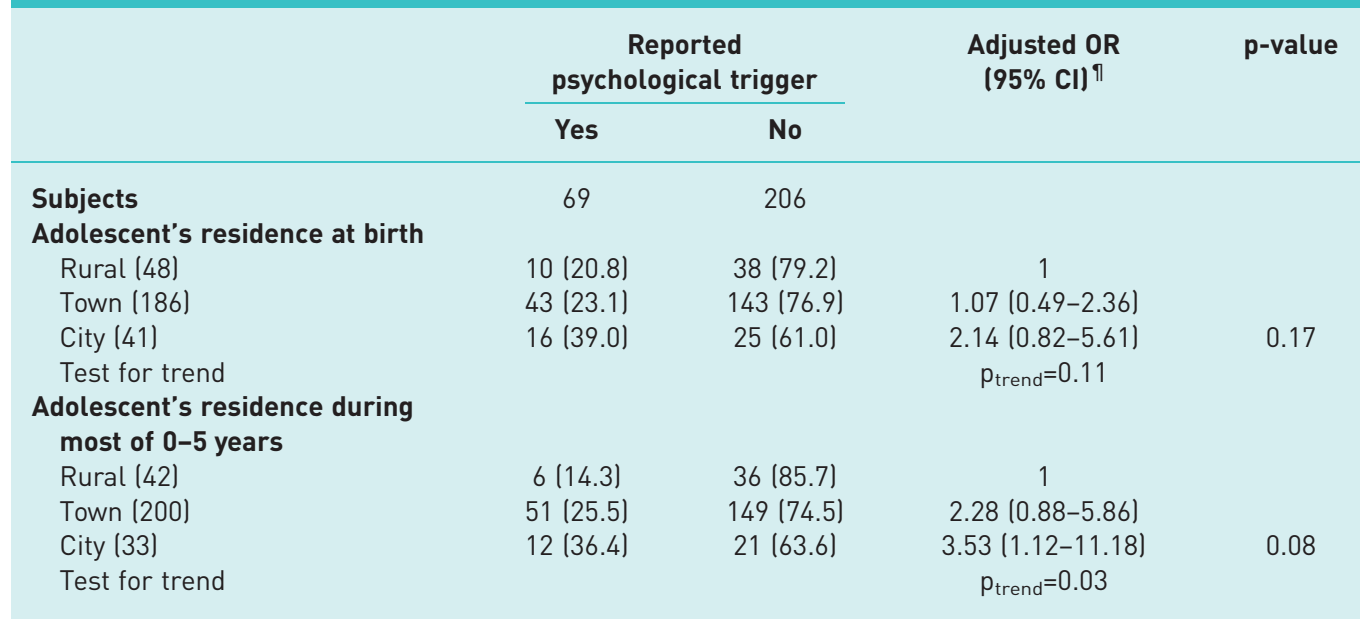

Data are presented as $n$ or $n(\%)$, unless otherwise stated. " $: n=275$; ${ }^{\natural}$ : adjusted for child's age, sex and father's highest education level. 
lifestyle and socioeconomic factors have stronger overall effects than infrastructure factors $[9,28]$. Indeed, there is increasing evidence for adverse effects of exposure to psychosocial factors in early life on increased risk of both asthma and anxiety disorders in later childhood: several studies have found that maternal anxiety disorders and parenting difficulties were positively associated with anxiety disorders in childhood $[11,29,30]$, and that maternal prenatal/postnatal psychosocial distress and parenting difficulties were associated with increased risk of asthma (and allergy-related diseases) in childhood [11, 31-34]. Most studies investigating the adverse role of parental psychosocial distress on childhood asthma have been conducted in high-income countries, with only one study from sub-Saharan Africa that was conducted in South Africa [35]. It is important to investigate whether psychosocial distress or parenting difficulties are higher among urban dwellers than their rural counterparts in LMICs and whether this could possibly contribute to the observed increased prevalence of asthma in urban areas. Moreover, some studies have reported a positive association between anxiety disorders and urbanicity [36, 37], and between anxiety disorders and higher [38] or lower parental education [39]. However, there is a scarcity of such data from sub-Saharan Africa, and such information would be vital in informing the design of intervention studies for the primary and secondary prevention of both anxiety disorders and asthma among children.

The positive association between anxiety disorders and poor asthma control has been reported [40, 41]. Since there was no follow-up in this study, temporality cannot be assumed. Nonetheless, we previously observed that a quarter of adolescents with asthma (in the parent case-control study) reported that their symptoms were triggered by psychological factors (such as psychological distress and laughter) [27]. These findings emphasise the need to manage the two conditions concurrently. This may include the routine screening of asthmatic adolescents for anxiety disorders and the proper management of the latter [42]. We observed a positive association between self-reported allergy-related diseases and anxiety disorders, but a lack of association between SPTs and anxiety disorders. This discrepancy may be explained by either self-report bias or that the positive association seen with self-reported allergy-related diseases may be mediated through non-atopic mechanisms.

There are several limitations to this study. The parent study was designed as a case-control study and therefore asthmatic individuals were over-represented compared with a general population. We cannot estimate the population prevalence of anxiety disorders. There were no data on current residence (although adolescents were attending schools in urban Uganda, it is possible the rural-born adolescents return to rural areas during the school holiday and urban-born adolescents return to urban areas), to control for any potential bias related to differential enrolment of adolescents born in urban and rural Uganda. Another limitation was the relatively small sample size, which meant that we had reduced power for some analyses. Nevertheless, within the small group of adolescents, we have been able to contribute data from Africa on the relationship between anxiety disorders and asthma among adolescents. We also make the intriguing observation of a positive trend towards adolescents who reported psychological distress/excitement as a trigger for asthma being more likely to have been raised in urban than rural areas. More studies will expand our understanding of the relationship between anxiety disorders and asthma.

\section{Conclusions}

We confirm a positive association between anxiety disorders and asthma among adolescents in urban Uganda. In addition, we demonstrate a close similarity in early-life risk factors associated with both anxiety disorders and with asthma among adolescents, suggesting shared underlying environmental exposures. This also implies that looking closely at the risk factors for anxiety disorders will provide more insight into the risk factors for asthma. More research is required to identify the adverse environmental factors in the urban areas in LMICs that increase both anxiety disorders and asthma, to assess underlying mechanisms, and to perform intervention studies for prevention.

Acknowledgements: We extend our gratitude to the large team of researchers at the Medical Research Council/Uganda Virus Research Institute and London School of Hygiene and Tropical Medicine Uganda Research Unit (Entebbe, Uganda) involved in the data collection and management for this study. We thank the study participants, their parents, and the school and education authorities for their enthusiastic participation and contribution towards the success of this study.

Author contributions: H. Mpairwe made substantial contributions to the conception and design, the acquisition, analysis and interpretation of data, and drafting the work and revising it critically for important intellectual content. R.S. Mpango made substantial contributions to the acquisition and interpretation of data, and drafting the work and revising it critically for important intellectual content. W. Sembajiwe and E.L. Webb made substantial contributions to the analysis and interpretation of data, and revising the work critically for important intellectual content. A.M. Elliott, N. Pearce and E. Kinyanda made substantial contributions to the conception and design, the interpretation of data, and revising the work critically for important intellectual content. All authors gave the final approval of the version to be published and are in agreement to be accountable for all aspects of the work in ensuring that questions related to the accuracy or integrity of any part are appropriately investigated and resolved. 
Support statement: This work was funded by Wellcome (reference 102512, training fellowship to H. Mpairwe; 204928/Z/ 16/Z Institutional Strategic Support Fund to H. Mpairwe; 095778 senior fellowship to A.M. Elliott) and a Medical Research Council/Dept for International Development African Leadership Award (to E. Kinyanda, reference MR/ L004623/1). This work was partly funded by the European Research Council under the European Union's Seventh Framework Programme (FP7/2007-2013)/ERC grant (to N. Pearce, agreement 668954). Funding information for this article has been deposited with the Crossref Funder Registry.

Conflict of interest: None declared.

\section{References}

1 World Health Organization. Adolescent mental health: key facts. 2019. www.who.int/news-room/fact-sheets/detail/ adolescent-mental-health Date last accessed: 12 March 2021.

2 Strawn JR, Lu L, Peris TS, et al. Research Review: Pediatric anxiety disorders - what have we learnt in the last 10 years? J Child Psychol Psychiatr 2020; 62: 114-139.

3 Kessler RC, Angermeyer M, Anthony JC, et al. Lifetime prevalence and age-of-onset distributions of mental disorders in the World Health Organization's World Mental Health Survey Initiative. World Psychiatry 2007; 6 : 168-176

4 World Health Organization. Asthma: key facts. 2020. www.who.int/news-room/fact-sheets/detail/asthma Date last accessed: 12 March 2021.

5 Castro-Rodriguez JA, Forno E, Rodriguez-Martinez CE, et al. Risk and protective factors for childhood asthma: what is the evidence? J Allergy Clin Immunol Pract 2016; 4: 1111-1122.

6 Asher MI, Montefort S, Bjorksten B, et al. Worldwide time trends in the prevalence of symptoms of asthma, allergic rhinoconjunctivitis, and eczema in childhood: ISAAC Phases One and Three repeat multicountry cross-sectional surveys. Lancet 2006; 368: 733-743.

7 Kirenga BJ, de Jong C, Katagira W, et al. Prevalence and factors associated with asthma among adolescents and adults in Uganda: a general population based survey. BMC Public Health 2019; 19: 227.

8 Shimwela M, Mwita JC, Mwandri M, et al. Asthma prevalence, knowledge, and perceptions among secondary school pupils in rural and urban coastal districts in Tanzania. BMC Public Health 2014; 14: 387.

9 Rodriguez A, Brickley E, Rodrigues L, et al. Urbanisation and asthma in low-income and middle-income countries: a systematic review of the urban-rural differences in asthma prevalence. Thorax 2019; 74: 1020-1030.

10 Mpairwe H, Namutebi M, Nkurunungi G, et al. Risk factors for asthma among schoolchildren who participated in a case-control study in urban Uganda. eLife 2019; 8: e49496.

11 Klinnert MD, Nelson HS, Price MR, et al. Onset and persistence of childhood asthma: predictors from infancy. Pediatrics 2001; 108: e69.

12 Katon WJ, Richardson L, Lozano P, et al. The relationship of asthma and anxiety disorders. Psychosom Med 2004 66: 349-355

13 Blackman JA, Gurka MJ. Developmental and behavioral comorbidities of asthma in children. J Dev Behav Pediatr 2007; 28: 92-99.

14 Hasler G, Gergen PJ, Kleinbaum DG, et al. Asthma and panic in young adults: a 20-year prospective community study. Am J Respir Crit Care Med 2005; 171: 1224-1230.

15 Chida Y, Hamer M, Steptoe A. A bidirectional relationship between psychosocial factors and atopic disorders: a systematic review and meta-analysis. Psychosom Med 2008; 70: 102-116.

16 Brew BK, Lundholm C, Gong T, et al. The familial aggregation of atopic diseases and depression or anxiety in children. Clin Exp Allergy 2018; 48: 703-711.

17 Harriet M, Webb EL. SONA Project - anxiety disorders and asthma dataset. 2020. https://datacompass.lshtm.ac uk/id/eprint/1893 Date last accessed: 12 March 2021.

18 von Elm E, Altman DG, Egger M, et al. The Strengthening the Reporting of Observational Studies in Epidemiology (STROBE) statement: guidelines for reporting observational studies. Int J Surg 2014; 12: 1495-1499.

19 World Health Organization. Adolescent health. 2020. www.who.int/health-topics/adolescent-health Date last accessed: 12 March 2021.

20 Gadow KD, Sprafkin J. The Youth's Inventory-4 (YI-4). 2016. www.checkmateplus.com/product/yi-4.htm Date last accessed: 12 March 2021.

21 Kinyanda E, Salisbury TT, Levin J, et al. Rates, types and co-occurrence of emotional and behavioural disorders among perinatally HIV-infected youth in Uganda: the CHAKA study. Soc Psychiatry Psychiatr Epidemiol 2019; 54 415-425.

22 Asher MI, Keil U, Anderson HR, et al. International Study of Asthma and Allergies in Childhood (ISAAC) rationale and methods. Eur Respir J 1995; 8: 483-491.

23 Liu AH, Zeiger R, Sorkness C, et al. Development and cross-sectional validation of the Childhood Asthma Control Test. J Allergy Clin Immunol 2007; 119: 817-825.

24 Nathan RA, Sorkness CA, Kosinski M, et al. Development of the asthma control test: a survey for assessing asthma control. J Allergy Clin Immunol 2004; 113: 59-65.

25 Heinzerling L, Mari A, Bergmann KC, et al. The skin prick test - European standards. Clin Transl Allergy 2013; 3: 3.

26 Aaro LE, Flisher AJ, Kaaya S, et al. Parental education as an indicator of socioeconomic status: improving quality of data by requiring consistency across measurement occasions. Scand J Public Health 2009; 37: Suppl. 2, 16-27.

27 Mpairwe H, Tumwesige P, Namutebi M, et al. Asthma control and management among schoolchildren in urban Uganda: results from a cross-sectional study. Wellcome Open Res 2019; 4: 168.

28 Rodriguez A, Vaca M, Oviedo G, et al. Urbanisation is associated with prevalence of childhood asthma in diverse, small rural communities in Ecuador. Thorax 2011; 66: 1043-1050.

29 McClure EB, Brennan PA, Hammen C, et al. Parental anxiety disorders, child anxiety disorders, and the perceived parent-child relationship in an Australian high-risk sample. J Abnorm Child Psychol 2001; 29: 1-10.

30 Aktar E, Nikolić M, Bögels SM. Environmental transmission of generalized anxiety disorder from parents to children: worries, experiential avoidance, and intolerance of uncertainty. Dialogues Clin Neurosci 2017; 19 : 137-147. 
van de Loo KF, van Gelder MM, Roukema J, et al. Prenatal maternal psychological stress and childhood asthma and wheezing: a meta-analysis. Eur Respir J 2016; 47: 133-146.

32 Brew BK, Lundholm C, Viktorin A, et al. Longitudinal depression or anxiety in mothers and offspring asthma: a Swedish population-based study. Int J Epidemiol 2018; 47: 166-174.

33 Kozyrskyj AL, Mai XM, McGrath P, et al. Continued exposure to maternal distress in early life is associated with an increased risk of childhood asthma. Am J Respir Crit Care Med 2008; 177: 142-147.

34 Pierce M, Hope HF, Kolade A, et al. Effects of parental mental illness on children's physical health: systematic review and meta-analysis. Br J Psychiatry 2019; 217: 354-363.

35 MacGinty RP, Lesosky M, Barnett W, et al. Associations between maternal mental health and early child wheezing in a South African birth cohort. Pediatr Pulmonol 2018; 53: 741-754.

36 Peen J, Schoevers RA, Beekman AT, et al. The current status of urban-rural differences in psychiatric disorders. Acta Psychiatr Scand 2010; 121: 84-93.

37 Hyland P, Shevlin M, Elklit A, et al. Social, familial and psychological risk factors for mood and anxiety disorders in childhood and early adulthood: a birth cohort study using the Danish Registry System. Soc Psychiatry Psychiatr Epidemiol 2016; 51: 331-338.

38 Ochi M, Fujiwara T, Mizuki R, et al. Association of socioeconomic status in childhood with major depression and generalized anxiety disorder: results from the World Mental Health Japan survey 2002-2006. BMC Public Health 2014; 14: 359.

39 McLaughlin KA, Breslau J, Green JG, et al. Childhood socio-economic status and the onset, persistence, and severity of DSM-IV mental disorders in a US national sample. Soc Sci Med 2011; 73: 1088-1096.

40 Sastre J, Crespo A, Fernandez-Sanchez A, et al. Anxiety, depression, and asthma control: changes after standardized treatment. J Allergy Clin Immunol Pract 2018; 6: 1953-1959.

41 Ciprandi G, Schiavetti I, Rindone E, et al. The impact of anxiety and depression on outpatients with asthma. Ann Allergy Asthma Immunol 2015; 115: 408-414.

42 McGovern C, Arcoleo K, Melnyk B. Sustained effects from a school-based intervention pilot study for children with asthma and anxiety. J Sch Nurs 2020; in press [https://doi.org/10.1177/1059840520934178]. 